\title{
A NOTE ON SMOOTH FORMS ON ANALYTIC SPACES
}

\author{
MATS ANDERSSON \& HÅKAN SAMUELSSON KALM
}

Abstract. We prove that any smooth mapping between reduced analytic spaces induces a natural pullback operation on smooth differential forms.

\section{INTRODUCTION}

There is a natural notion of smooth differential forms on any reduced analytic space. The dual objects are the currents. Such forms and currents have turned out to be useful tools, e.g., in [4, 5, 7], in the analytic approach to intersection theory [2, 3], and in the context of the $\bar{\partial}$-equation on analytic spaces [1, 8].

It is desirable to be able to take the direct image of a current under a proper map $f: X \rightarrow Z$ between reduced analytic spaces. By duality this amounts to take pullbacks of smooth forms. In some works, e.g., [2, 3], it is implicitly assumed that this is possible. There is an obvious tentative definition of $f^{*} \phi$ for a smooth form $\phi$ on $Z$. It is however not clear that it gives a well-defined pullback operation, not even if $f$ and $\phi$ are holomorphic and $\phi$ has positive degree; this case is settled in [5. Corollary 1.0.2]. The main problem is when $f$ is the inclusion of an analytic subvariety contained in $Z_{\text {sing }}$. It was proved in [4, III Corollary 2.4.11] that if $f$ is holomorphic, then the suggested definition indeed gives a functorial operation on smooth forms. In this short note we give a new proof of this fact. Moreover, we extend it to the case when $f$ is merely smooth, see Theorem 2.1 below. Our result is implicitly claimed in [6], see Remark 2.2 below.

Acknowledgment: We are grateful to the referee for careful reading and important comments.

\section{Results}

Let $X$ be a reduced analytic space. Recall that, by definition, there is a neighborhood $U$ of any point in $X$ and an embedding $i: U \rightarrow D$ in an open set $D \subset \mathbb{C}^{N}$ such that $U$ can be identified with its image. For notational convenience we will suppress $U$ and say that $i$ is a local embedding of $X$. A smooth $(p, q)$-form $\phi$ on $X_{\text {reg }}$ is smooth on $X, \phi \in \mathcal{E}^{p, q}(X)$, if there is a smooth form $\varphi$ in $D$ such that

$$
\left.i\right|_{X_{r e g}} ^{*} \varphi=\phi .
$$

If $j: X \rightarrow D^{\prime}$ is another local embedding, then the identity on $X$ induces a biholomorphism $i(X) \stackrel{\sim}{\rightarrow} j(X)$. Thus, again by definition, locally in $D$ and $D^{\prime}$, there are holomorphic maps $g: D \rightarrow D^{\prime}$ and $h: D^{\prime} \rightarrow D$ such that $i=h \circ j$ and $j=g \circ i$. Since $h^{*} \varphi$ is smooth in $D^{\prime}$ and

$$
\left.j\right|_{X_{\text {reg }}} ^{*} h^{*} \varphi=\phi
$$

\footnotetext{
Date: July 8, 2020.

${ }^{1}$ By an embedding we always mean a closed holomorphic embedding so that the image is an analytic subvariety.
} 
it follows that the notion of smooth forms on $X$ is independent of embedding.

We will write $i^{*} \varphi$ for the image of $\varphi \in \mathcal{E}(D)$ in $\mathcal{E}(X)$. Let $[i(X)]$ be the Lelong current of integration over $i(X)_{\text {reg. }}$. The kernel of $i^{*}$ is closed since

$$
i^{*} \varphi=0 \Longleftrightarrow \varphi \wedge[i(X)]=0 .
$$

Thus, with the quotient topology $\mathcal{E}(X)=\mathcal{E}(D) / \operatorname{Ker} i^{*}$ is a Fréchet space. To see that this topology is independent of the embedding, notice that it is defined by the semi-norms

$$
|\phi|_{X, i}:=\inf \left\{|\varphi|_{D} ; i^{*} \varphi=\phi\right\},
$$

where $|\cdot|_{D}$ are the semi-norms defining the topology on $\mathcal{E}(D)$. Let $j, g$, and $h$ be as above and let $|\cdot|_{X, j}$ be the analogously defined semi-norms induced by $j$. Since $j^{*} \psi=\phi$ implies that $i^{*} g^{*} \psi=\phi$ and since $g^{*}: \mathcal{E}\left(D^{\prime}\right) \rightarrow \mathcal{E}(D)$ is continuous we get

$$
|\phi|_{X, i}=\inf _{i^{*} \varphi=\phi}|\varphi|_{D} \leq \inf _{j^{*} \psi=\phi}\left|g^{*} \psi\right|_{D} \leq C \inf _{j^{*} \psi=\phi}|\psi|_{D^{\prime}}=C|\phi|_{X, j} .
$$

In the same way, $|\phi|_{X, j} \leq C^{\prime}|\phi|_{X, i}$ and it follows that the semi-norms $|\phi|_{X, j}$ give the same topology as $|\phi|_{X, i}$.

Let $\mathcal{E}_{X}^{p, q}$ be the sheaf of smooth $(p, q)$-forms on $X$ and let $\mathcal{E}_{X}^{r}=\oplus_{p+q=r} \mathcal{E}_{X}^{p, q}$. We say that a continuous map $f: X \rightarrow Z$ between reduced analytic spaces is smooth if $f^{*} \phi \in \mathcal{E}_{X}^{0}$ for any $\phi \in \mathcal{E}_{Z}^{0}$. Notice that if $i: X \rightarrow D_{X}$ and $j: Z \rightarrow D_{Z}$ are local embeddings, then $f$ is the restriction to $i(X)$ of a smooth map $D_{X} \rightarrow D_{Z}$.

Theorem 2.1. Let $f: X \rightarrow Z$ be a smooth map between reduced analytic spaces. There is a well-defined map $f^{*}: \mathcal{E}^{r}(Z) \rightarrow \mathcal{E}^{r}(X)$ with the following property: If $\phi$ is a smooth form on $Z, i: X \rightarrow D_{X}$ and $\iota: Z \rightarrow D_{Z}$ are local embeddings, $\varphi$ is a smooth form in $D_{Z}$ such that $\iota^{*} \varphi=\phi$, and $\tilde{f}: D_{X} \rightarrow D_{Z}$ is a smooth map such that $\left.\tilde{f}\right|_{i(X)}=f$, then

$$
f^{*} \phi=i^{*} \tilde{f}^{*} \varphi
$$

Assume that $g: X \rightarrow Y$ and $h: Y \rightarrow Z$ are smooth maps such that $f=h \circ g$. Let $j: Y \rightarrow D_{Y}$ be a local embedding and $\tilde{g}: D_{X} \rightarrow D_{Y}$ and $\tilde{h}: D_{Y} \rightarrow D_{Z}$ smooth maps such that $\left.\tilde{g}\right|_{i(X)}=g$ and $\left.\tilde{h}\right|_{j(Y)}=h$, respectively. Notice that the restriction of $\tilde{h} \circ \tilde{g}$ to $i(X)$ is $f$. If $\phi \in \mathcal{E}(Z)$ and $\phi=\iota^{*} \varphi$ it follows by Theorem 2.1 that $h^{*} \phi=j^{*} \tilde{h}^{*} \varphi$ and $g^{*} h^{*} \phi=i^{*} \tilde{g}^{*} \tilde{h}^{*} \varphi=f^{*} \phi$. Hence,

$$
f^{*} \phi=g^{*} h^{*} \phi, \quad \phi \in \mathcal{E}(Z) .
$$

Remark 2.2. An a priori different definition of smooth forms on $X$ is given in $[6$, Section 3.3]. If $i: X \rightarrow D$ is a local embedding, then the space of smooth forms on $X$ is defined in [6, Section 3.3] as $\mathcal{E}(D) / \mathcal{N}(D)$, where $\mathcal{N}(D)$ is the space of smooth forms $\varphi$ in $D$ such that for any smooth manifold $W$ and any smooth map $g: W \rightarrow D$ with $g(W) \subset X$ one has $g^{*} \varphi=0$.

It is clear that $\mathcal{N}(D) \subset \operatorname{Ker} i^{*}$ and it is in fact claimed in [6, Section 3.3] that $\mathcal{N}(D)=\operatorname{Ker} i^{*}$, but we have not been able to find a proof in the literature. It follows from Theorem 2.1 that the claim indeed is true: If $g: W \rightarrow D$ is a smooth map with $g(W) \subset X$, then $g=i \circ \gamma$ for a smooth map $\gamma: W \rightarrow X$. In view of (2.2) thus $g^{*} \varphi=\gamma^{*} i^{*} \varphi=0$ if $i^{*} \varphi=0$.

The space of currents on $X, \mathscr{C}(X)$, is the dual of the space of test forms, i.e., compactly supported forms in $\mathcal{E}(X)$, cf. [7, Section 4.2]. Let $f: X \rightarrow Z$ be as in 
Theorem 2.1 and assume that $f$ is proper. Then $f^{*} \phi$ is a test form on $X$ if $\phi$ is a test form on $Z$. If $\mu$ is a current on $X$ thus $f_{*} \mu$ is a current on $Z$ defined by

$$
f_{*} \mu . \phi=\mu . f^{*} \phi .
$$

By Theorem 2.1 and (2.2) we get

Corollary 2.3. Let $f: X \rightarrow Z$ be a smooth proper map between reduced analytic spaces. Then the induced mapping $f_{*}: \mathscr{C}(X) \rightarrow \mathscr{C}(Z)$ has the property that if $f=$ $h \circ g$, where $g: X \rightarrow Y$ and $h: Y \rightarrow Z$ are smooth proper maps, then

$$
f_{*} \mu=h_{*} g_{*} \mu, \quad \mu \in \mathscr{C}(X) .
$$

Example 2.4. Suppose that $i: X \rightarrow D$ is an embedding and consider the induced mapping $i_{*}: \mathscr{C}(X) \rightarrow \mathscr{C}(D)$. It follows from (2.3) and the definition of test forms on $X$ that $i_{*}$ is injective. Thus $\mathscr{C}(X)$ can be identified with its image $i_{*} \mathscr{C}(X)$. In view of the definition of $\mathscr{C}(X)$ and (2.3) it follows that $i_{*} \mathscr{C}(X)$ is the set of currents $\mu$ in $D$ such that $\mu \cdot \varphi=0$ if $i^{*} \varphi=0$. Notice in particular that $i_{*} 1=[i(X)]$.

\section{Proofs}

We will prove Theorem 2.1 by showing that the right-hand side of (2.1) is independent of the choices of embeddings $i, \iota$ and extensions $\tilde{f}$ and $\varphi$ of $f$ and $\phi$, respectively. The technical part is contained in Proposition 3.2, cf. [4, Proposition III 2.4.10] and [5. Proposition 1.0.1]. We begin with the following lemma.

Lemma 3.1. Let $M$ be a reduced analytic space, $N$ a complex manifold, and $p: M \rightarrow$ $N$ a proper holomorphic map. If $\operatorname{dim} N=d \geq 1$ and $\operatorname{rank}_{x} p<d$ for all $x \in M_{\text {reg }}$, then $p$ is not surjective.

Proof. If $M$ is smooth it follows from the constant rank theorem that $p$ cannot be surjective. If $M$ is not smooth, let $\pi: \widetilde{M} \rightarrow M$ be a Hironaka resolution of singularities. Then $\widetilde{M}$ is smooth and $\tilde{p}:=p \circ \pi$ is a proper holomorphic map with the same image as $p$. Since $\pi$ is a biholomorphism outside the exceptional divisor $E=\pi^{-1}\left(M_{\text {sing }}\right)$ we have $\operatorname{rank}_{x} \tilde{p}<d$ for all $x \in \widetilde{M} \backslash E$. By semi-continuity of the rank it follows that $\operatorname{rank}_{x} \tilde{p}<d$ for all $x \in \widetilde{M}$. By the constant rank theorem thus $\tilde{p}$ cannot be surjective.

Proposition 3.2. Let $D \subset \mathbb{C}^{N}$ be an open set and let $\varphi$ be a smooth form in $D$.

(i) Let $W \subset V$ be analytic subsets of $D$. If the pullback of $\varphi$ to $V_{\text {reg }}$ vanishes, then the pullback of $\varphi$ to $W_{\text {reg }}$ vanishes.

(ii) Let $W$ be a smooth not necessarily complex submanifold of $D$, let $V \subset D$ be an analytic subset, and assume that $W \subset V$. If the pullback of $\varphi$ to $V_{\text {reg }}$ vanishes, then the pullback of $\varphi$ to $W$ vanishes.

Proof of part (i). We may assume that $W$ is irreducible of dimension $d$. We may also assume that $\varphi$ has positive degree since a smooth function vanishing on $V_{\text {reg }}$ must vanish on $W$ by continuity. The case $d=0$ is then clear since the pullback of a form of positive degree to discrete points necessarily vanishes. Let $\tilde{\pi}: V^{\prime} \rightarrow V$ be a Hironaka resolution of singularities. Suppose that $W^{\prime} \subset V^{\prime}$ is analytic and such that $\tilde{\pi}\left(W^{\prime}\right)=W$. Let $\pi=\left.\tilde{\pi}\right|_{W^{\prime}}$ and let $\phi$ be the pullback of $\varphi$ to $W_{\text {reg }}$. Since the pullback of $\varphi$ under $W^{\prime} \hookrightarrow V^{\prime} \rightarrow V \hookrightarrow D$ is 0 , it follows that $\pi^{*} \phi=0$. We will find such $W^{\prime}$ and $\pi$ such that $\pi^{*} \phi=0$ implies $\phi=0$. 
To begin with we set $W^{\prime}=\tilde{\pi}^{-1}(W)$. If $\tilde{\pi}\left(W_{\text {sing }}^{\prime}\right)=W$, replace $W^{\prime}$ by $W_{\text {sing }}^{\prime}$. Possibly repeating this we may assume that $\tilde{\pi}\left(W_{\text {sing }}^{\prime}\right) \nsubseteq W$. Thus $\tilde{\pi}\left(W_{\text {sing }}^{\prime}\right)$ is a proper analytic subset of $W$. Set $\pi=\left.\tilde{\pi}\right|_{W^{\prime}}$ and notice that $\pi: W^{\prime} \rightarrow W$ is proper and surjective.

Let

$$
M=W^{\prime} \backslash \pi^{-1}\left(W_{\text {sing }} \cup \pi\left(W_{\text {sing }}^{\prime}\right)\right), \quad N=W_{\text {reg }} \backslash \pi\left(W_{\text {sing }}^{\prime}\right),
$$

and let $p=\left.\pi\right|_{M}$. Since $M$ is smooth and $p: M \rightarrow N$ is proper and surjective it follows from the constant rank theorem that there is $x \in M$ such that $\operatorname{rank}_{x} p=d$. Since $d$ is the optimal rank of $p$ this holds for $x$ in a non-empty Zariski-open subset of $M$. Let $\widetilde{M}=\left\{x \in M ; \operatorname{rank}_{x} p \leq d-1\right\}$ be the complement of this set. Then $\left.\operatorname{rank}_{x} p\right|_{\widetilde{M}} \leq d-1$ for all $x \in \widetilde{M}_{\text {reg }}$. By Lemma 3.1, $p(\widetilde{M}) \nsubseteq N$ and thus $p(\widetilde{M})$ is a proper analytic subset of $N$.

Now, $N \backslash p(\widetilde{M})$ is a dense open subset of $W_{\text {reg }}$ and so it suffices to show that $\phi=0$ there. However, $M \backslash p^{-1} p(\widetilde{M})$ is a (non-empty) open subset of $M$ and in this set $p$ has constant rank $=d=\operatorname{dim} W$. Thus, $p$ is locally a simple projection and it follows that if $p^{*} \phi=0$, then $\phi=0$.

Proof of part (ii). We use induction over $\operatorname{dim} V$. The case $\operatorname{dim} V=0$ is clear so suppose that $\operatorname{dim} V>0$.

Take a point $w \in W$. If $w \in V_{\text {reg }}$, then there is a neighborhood $U \subset W$ of $w$ contained in $V_{\text {reg }}$. Then clearly the pullback of $\varphi$ to $U$ vanishes. Assume now that $w \in V_{\text {sing. }}$. If there is a neighborhood $U \subset W$ of $w$ contained in $V_{\text {sing }}$, then the pullback of $\varphi$ to $U$ vanishes in view of the induction hypothesis and part (i) of this proposition. If not, then there is a sequence of points $w_{j} \in W$ converging to $w$ such that $w_{j} \in V_{\text {reg. }}$. Then there are neighborhoods $U_{j} \subset W$ of $w_{j}$ contained in $V_{\text {reg }}$. The pullback of $\varphi$ to $U_{j}$ vanish. Now, $\varphi(w)$ is a multilinear mapping on $T_{w} D$ depending continuously on $w$. Since the pullback of $\varphi$ to $U_{j}$ vanish the restriction of $\varphi\left(w_{j}\right)$ to $T_{w_{j}} W$ vanish. By continuity thus the restriction of $\varphi(w)$ to $T_{w} W$ vanishes.

Hence, for any $w \in W$, the restriction of $\varphi(w)$ to $T_{w} W$ vanishes; thus the pullback of $\varphi$ to $W$ vanishes.

Proof of Theorem 2.1. Let $\phi \in \mathcal{E}(Z)$ and let $f^{*} \phi$ be the form on $X_{\text {reg }}$ defined by the right-hand side of (2.1). Clearly $f^{*} \phi$ is smooth on $X$. As mentioned, we will show that it is independent of the choices of extensions $\tilde{f}$ and $\varphi$ as well as of the local embeddings.

First assume that $X$ is smooth. The set $X_{1} \subset X$ of points where $f$ has maximal rank is open. By the constant rank theorem each point $x \in X_{1}$ has a neighborhood $U_{x}$ such that $\left.f\right|_{U_{x}}$ is a submersion onto a smooth submanifold $f\left(U_{x}\right)$ of $D_{Z}$ contained in $Z$. By Proposition 3.2 (ii), if $\varphi$ is a smooth form in $D_{Z}$ such that $\iota^{*} \varphi=\phi$, then the pullback of $\varphi$ to $f\left(U_{x}\right)$ only depends on the pullback of $\varphi$ to $Z_{\text {reg }}$, i.e., only on $\phi$. Thus, $f^{*} \phi$ is well-defined in $U_{x}$. Hence, $f^{*} \phi$ is well-defined in $X_{1}$ and so, by continuity, well-defined in the closure $\bar{X}_{1}$. Repeating the argument with $X$ and $f$ replaced by $X \backslash \bar{X}_{1}$ and $\left.f\right|_{X \backslash \bar{X}_{1}}$ it follows that $f^{*} \phi$ is well-defined in $\bar{X}_{2}$, where $X_{2} \subset X \backslash \bar{X}_{1}$ is the set of points where $\left.f\right|_{X \backslash \bar{X}_{1}}$ has maximal rank. Notice that this rank is strictly less than the rank of $f$ in $X_{1}$. Thus, continuing the process of constructing such open sets $X_{k}$, after a finite number of steps we get $X_{k}=\emptyset$. Since $X=\cup_{j} \bar{X}_{j}, f^{*} \phi$ is well-defined in $X$. 
In the case of a general $X$ we restrict $f$ to $X_{\text {reg }}$ and conclude that $f^{*} \phi$ is welldefined on $X_{r e g}$, which by definition means that $f^{*} \phi$ is well-defined.

\section{REFERENCES}

[1] Andersson, Mats; Samuelsson HÅkAn A Dolbeault-Grothendieck lemma on complex spaces via Koppelman formulas. Invent. Math. 190 (2012), 261-297.

[2] Andersson, Mats; Samuelsson Kalm, Håkan; Wulcan, Elizabeth; Yger, Alain Segre numbers, a generalized King formula, and local intersections. J. Reine Angew. Math. 728 (2017), $105-136$.

[3] Andersson, Mats; Eriksson, Dennis; Samuelsson Kalm, Håkan; Wulcan, Elizabeth; YGER, AlAin Global representation of Segre numbers by Monge-Ampère products. Math. Ann., to appear. Available at arXiv:1812.03054

[4] Barlet, Daniel; Magnússon, Jón Cycles analytiques complexes. I. Théorèmes de préparation des cycles. Cours Spécialisés, 22. Société Mathématique de France, Paris, 2014. 525 pp.

[5] Barlet, Daniel The sheaf $\alpha_{X}^{\bullet}$. J. Singul. 18 (2018), 50-83.

[6] Bloom, Thomas; Herrera, Miguel De Rham cohomology of an analytic space. Invent. Math. 7 (1969), 275-296.

[7] Herrera, M.; Lieberman, D. Residues and principal values on complex spaces. Math. Ann. 194 (1971), 259-294

[8] Ruppenthal, Jean; Samuelsson Kalm, Håkan; Wulcan, Elizabeth Explicit Serre duality on complex spaces. Adv. Math. 305 (2017), 1320-1355.

M. Andersson, H. Samuelsson Kalm, Department of Mathematical Sciences, Division of Algebra and Geometry, University of Gothenburg and Chalmers University of Technology, SE-412 96 Göteborg, Sweden

E-mail address: matsa@chalmers.se, hasam@chalmers.se 